\title{
Mean nocturnal respiratory rate predicts cardiovascular and all-cause mortality in community-dwelling older men and women
}

\author{
Mathias Baumert ${ }^{1}$, Dominik Linz ${ }^{2}$, Katie Stone ${ }^{3}$, R. Doug McEvoy ${ }^{4}$, \\ Steve Cummings ${ }^{3}$, Susan Redline ${ }^{5,6}$, Reena Mehra ${ }^{7}$ and Sarah Immanuel ${ }^{1,8}$
}

@ERSpublications

In community-dwelling older men and women, nocturnal respiratory rate of $\geqslant 16$ breaths $\mathrm{min}^{-1}$ is an independent predictor of long-term cardiovascular and all-cause mortality http://bit.ly/2XLzi0s

Cite this article as: Baumert $\mathrm{M}$, Linz $\mathrm{D}$, Stone $\mathrm{K}$, et al. Mean nocturnal respiratory rate predicts cardiovascular and all-cause mortality in community-dwelling older men and women. Eur Respir J 2019; 54: 1802175 [https://doi.org/10.1183/13993003.02175-2018].

ABSTRACT Respiratory frequency $(f \mathrm{R})$ predicts in-hospital and short-term mortality in patients with a variety of pathophysiological conditions, but its predictive value for long-term cardiovascular and all-cause mortality in the general population is unknown. Here, we investigated the relationship between mean nocturnal $f \mathrm{R}$ and mortality in community-dwelling older men and women.

We measured mean nocturnal $f \mathrm{R}$ during sleep from overnight polysomnography in 2686 men participating in the Osteoporotic Fractures in Men Study (MrOS) Sleep study and 406 women participating in the Study of Osteoporotic Fractures (SOF) to investigate the relationship between mean nocturnal $f \mathrm{R}$ and long-term cardiovascular and all-cause mortality.

$166(6.1 \%)$ men in the MrOS cohort (8.9 2.6 years' follow-up) and 46 (11.2\%) women in the SOF cohort (6.4 \pm 1.6 years' follow-up) died from cardiovascular disease. All-cause mortality was $51.2 \%$ and $26.1 \%$ during $13.7 \pm 3.7$ and $6.4 \pm 1.6$ years' follow-up in the MrOS Sleep study and the SOF cohorts, respectively. Multivariable Cox regression analysis adjusted for significant covariates demonstrated that $f \mathrm{R}$ dichotomised at 16 breaths. $\mathrm{min}^{-1}$ was independently associated with cardiovascular mortality (MrOS: hazard ratio (HR) 1.57, 95\% CI 1.14-2.15; p=0.005; SOF: HR 2.58, 95\% CI 1.41-4.76; $\mathrm{p}=0.002$ ) and all-cause mortality (MrOS: HR 1.18, 95\% CI 1.04-1.32; $\mathrm{p}=0.007$; SOF: HR 1.50, 95\% CI 1.02-2.20; $\mathrm{p}=0.04$ ).

In community-dwelling older men and women, polysomnography-derived mean nocturnal $f_{R}$ $\geqslant 16$ breaths. $\mathrm{min}^{-1}$ is an independent predictor of long-term cardiovascular and all-cause mortality. Whether nocturnal mean $f \mathrm{R}$ can be used as a risk marker warrants further prospective studies.

This article has supplementary material available from erj.ersjournals.com

All of the individual participant data generated during this study will be made available at the Osteoporotic Fractures in Men Study (MrOS) (https://mrosdata.sfcc-cpmc.net/) and the Study of Osteoporotic Fractures (https://sofonline.epi-ucsf. org/interface/) websites.

Received: Nov 142018 | Accepted after revision: April 212019

Copyright OERS 2019 


\section{Introduction}

Respiratory frequency $(f R)$ is one of the four vital signs, along with heart rate, arterial blood pressure and core temperature. In particular in older people [1], $f \mathrm{R}$ strongly predicts short-term in-hospital mortality $[2,3]$ in patients in the settings of pneumonia [4], trauma [5], stroke [6], cardiac arrest [7] and acute heart failure [8]. $f \mathrm{R}$ is typically measured by counting the number of breaths for 1 minute. In adults at rest, any $f \mathrm{R}$ between 12 and 20 breaths $\mathrm{min}^{-1}$ is considered normal; tachypnoea is defined as $f_{\mathrm{R}}>20$ breaths $\mathrm{min}^{-1}$ [9]. $f \mathrm{R}$ may increase with acute pathological conditions such as fever, in compensation for diabetic ketoacidosis or decompensated acute heart failure. Chronic pathophysiological conditions such as decompensated kidney disease, heart failure or chronic obstructive pulmonary disease (COPD) may also result in $f R$ changes.

While elevated blood pressure [10] and increased heart rate [11] are well-recognised risk factors for long-term cardiovascular morbidity and mortality in the general population, the association between $f \mathrm{R}$ and long-term cardiovascular outcomes is unclear. Dоммаsсн et al. [12] reported an association between nocturnal $f R$ and non-sudden cardiac death in survivors of myocardial infarction using Holter ECG and showed the independent predictive value of daytime $f \mathrm{R}$ for long-term outcomes in patients with acute myocardial infarction [13].

Here, we determined the prognostic value of $f \mathrm{R}$ for long-term cardiovascular and all-cause mortality in two large independent cohorts of community-dwelling older women and men. Since cardiorespiratory variables such as $f \mathrm{R}$ vary broadly across the day, adapting to changes in metabolic demand, emotional valences and arousal [14], we measured $f R$ during sleep, a state in which the body is in equilibrium, yielding highly standardised measurement conditions. We analysed $f R$ derived from overnight home-polysomnograms of 2686 older men participating in the Osteoporotic Fractures in Men Study (MrOS) Sleep study and 406 older women participating in the Study of Osteoporotic Fractures (SOF). We hypothesised that nocturnal mean $f_{R}$ predicts cardiovascular and all-cause mortality independent from established risk factors.

\section{Methods}

Study populations

MrOS sleep study

The MrOS was designed to describe the epidemiology of osteoporosis and fractures in older men, including the identification of risk factors for fracture and bone loss. Between March 2000 and April 2002, 5995 community-dwelling men aged $\geqslant 65$ years were enrolled [15]. Participants were recruited from six US centres. Subjects considered for enrolment in MrOS had to be able to walk without the assistance of another person and not have a bilateral hip replacement [16].

3135 men from the MrOS cohort were recruited for participation in the Outcomes of Sleep Disorders in Older Men (MrOS Sleep) (ClinicalTrials.gov identifier: NCT00070681). All men provided written informed consent, and the study was approved by the institutional review board at each site. The men were screened for use of mechanical devices during sleep including pressure mask for sleep apnoea, a mouthpiece for snoring or sleep apnoea or oxygen therapy. In general, those who reported the nightly use of any of these devices were excluded from the MrOS sleep study. However, 17 men who reported the use of one of these devices, but could forego use during the night of the sleep study were included. The 3135 men completed an exam conducted between December 2003 and March 2005 that included a clinic visit and overnight in-home polysomnography (PSG). Of these men, 2911 had technically adequate PSG.

\section{SOF}

The SOF is a multisite, prospective, observational study of community-dwelling women aged $\geqslant 65$ years and older [17]. The 9704 Caucasian participants constituting the original cohort were residents of four metropolitan areas in the USA (Baltimore, MD; Minneapolis, MN; Portland, OR; Pittsburgh/Monongahela

Affiliations: 'School of Electrical and Electronic Engineering, The University of Adelaide, Adelaide, Australia. ${ }^{2}$ Center for Heart Rhythm Disorders (CHRD), South Australian Health and Medical Research Institute (SAHMRI), University of Adelaide and Royal Adelaide Hospital, Adelaide, Australia. ${ }^{3}$ California Pacific Medical Center Research Institute, San Francisco, CA, USA. ${ }^{4}$ Adelaide Institute for Sleep Health, College of Medicine and Public Health, Flinders University, and Sleep Health Service, Respiratory and Sleep Services, Southern Adelaide Local Health Network, Adelaide, Australia. ${ }^{5}$ Division of Sleep and Circadian Disorders, Brigham and Women's Hospital and Harvard Medical School, Boston, MA, USA. ${ }^{6}$ Dept of Medicine, Beth Israel Deaconess Medical Center, Harvard Medical School, Boston, MA, USA. ${ }^{7}$ Center of Sleep Disorders, Neurologic Institute, Respiratory Institute, Heart and Vascular Institute, Lerner Research Institute, Cleveland Clinic, Cleveland, $\mathrm{OH}$, USA. ${ }^{8}$ College of Medicine and Public Health and Flinders Digital Health Research Centre, Flinders University, Adelaide, Australia.

Correspondence: Mathias Baumert, School of Electrical and Electronic Engineering, The University of Adelaide, SA 5005, Australia. E-mail: mathias.baumert@adelaide.edu.au 
Valley, PA). Initial enrolment took place between September 1986 and October 1988, and participants were reassessed at biannual follow-up visits. 662 African-American women recruited between February 1997 and February 1998 were added to the study.

As part of visit 8, which occurred between January 2002 and February 2004, unattended overnight 12-channel in-home PSG was completed in a convenience subset of 461 women recruited from two of the four clinical centres (Minnesota and Pittsburgh) [18].

\section{Follow-up}

MrOS sleep participants were surveyed for potential incident cardiovascular or clinically relevant arrhythmia events by postcard and/or phone contact every 4 months with $>99 \%$ response rate. Relevant medical records and supporting documentation from any potential incident clinical events were obtained by the clinical centre and forwarded to the coordinating centre for centralised adjudication by a board-certified cardiologist using a prespecified protocol, as previously described [19]. For fatal events, the death certificate and hospital records from the time of death were collected. For fatal events that occurred when participants were not hospitalised, a proxy interview with next of kin and hospital records from the most recent hospitalisation in the prior 12 months were obtained. Only events confirmed by the adjudicator are included for analysis.

SOF deaths were centrally adjudicated using a state-registered certificate of death which was submitted to the coordinating centre. The principal investigator at each of four clinical sites indicated the initial diagnosis for the cause of death. Final classification of cause-specific mortality was centrally adjudicated at the coordinating centre by a trained physician adjudicator, using the International Classification of Diseases 9th Revision Clinical Modification (ICD-9-CM).

\section{In-home overnight PSG}

Unattended PSG was performed over 1 night at the participant's residence using the Compumedics (Abbotsford, Australia) Safiro sleep monitoring system for MrOS Sleep and the Compumedics Siesta system for SOF.

Before the participant's bedtime, two trained staff members visited the participants to attach sensors and initiate data recording, including two central electroencephalograms, bilateral electrooculograms, bilateral chin electromyogram, a bipolar electrocardiogram, nasal-oral thermistor, nasal flow via pressure transducer and nasal cannula, abdominal and respiratory inductance plethysmography, finger pulse oximetry, bilateral leg movements by piezoelectric sensors and body position.

\section{Quantification of respiratory rate}

Overnight PSG comprising raw data stored in the European Data Format as well as scoring information stored in Compumedics output files in extensible markup language were retrieved for further processing within MATLAB environment using the signal processing toolbox. Breathing effort during sleep extracted from the thoracic respiratory inductance plethysmography belt signal of the PSG data was used for $f_{R}$ analysis during sleep. During pre-processing, signal offsets were removed and a low-pass forward and reverse Butterworth filter $(1 \mathrm{~Hz})$ was applied. Expiratory and inspiratory onsets were determined from the respiratory signal by identifying the peaks and valleys using the first-order derivative. The inspiratory onset of artifact-free breaths was used to compute a breath-by-breath measure of the respiratory interval which were then averaged within each subject. The mean respiratory interval of each subject was used to compute $f \mathrm{R}$ in breaths $\cdot \mathrm{min}^{-1}$.

\section{Other measures}

All participants completed a questionnaire at the time of the sleep visit, which included questions about medical history, specifically history of physician diagnosis of diabetes, hypertension and cardiovascular disease (coronary artery disease (CAD), myocardial infarction, stroke and heart failure, and sleep disordered breathing (SDB), quantified using the apnoea-hypopnoea index (AHI)). For MrOS participants, additional information was collected pertaining to COPD, chronic kidney disease and liver disease. In addition, participants were asked about their smoking status and alcohol use.

\section{Statistical analysis}

Values of $f \mathrm{R}$ were divided into quartiles for Kaplan-Meier curve survival analysis and log-rank testing. Anthropometric data, lifestyle metrics and medical history were compared using dichotomised variables and t-test and Chi-squared tests, respectively. Cox proportional hazard models were constructed for continuous and dichotomised variables. Dichotomisation was performed based on the highest quartile in the Kaplan-Meier curve. Adjustments were performed for those variables in table 1 that differed 
TABLE 1 Study cohort characteristics

\begin{tabular}{|c|c|c|c|c|c|c|c|c|}
\hline & \multicolumn{4}{|c|}{ MrOS } & \multicolumn{4}{|c|}{ SOF } \\
\hline & $f_{R}$ & $f_{R}<16$ breaths $\cdot \min ^{-1}$ & $f_{R} \geqslant 16$ breaths $\cdot \mathrm{min}^{-1}$ & $p$-value & $f_{R}$ & $f_{\mathrm{R}}<16$ breaths $\cdot \mathrm{min}^{-1}$ & $f_{R} \geqslant 16$ breaths $\cdot \min ^{-1}$ & $\overline{p \text {-value }}$ \\
\hline Subjects $n$ & 2686 & 1959 & 727 & & 406 & 241 & 165 & \\
\hline \multicolumn{9}{|l|}{ Anthropometric data } \\
\hline Age at PSG years & $76.2 \pm 5.4$ & $75.9 \pm 5.40$ & $77.0 \pm 5.64$ & $<0.001$ & $83.13 \pm 2.94$ & $82.9 \pm 2.74$ & $83.4 \pm 3.16$ & 0.05 \\
\hline \multicolumn{9}{|l|}{ Ethnicity/race } \\
\hline White & 2435 (90.7) & 1773 (90.5) & $662(91.1)$ & 0.51 & & $241(100)$ & $165(100)$ & \\
\hline African-American & 93 (3.5) & 64 (3.3) & $29(4.0)$ & & & & & \\
\hline Asian & $83(3.1)$ & $64(3.3)$ & $19(2.6)$ & & & & & \\
\hline Other & $75(2.8)$ & $58(3.0)$ & $17(2.3)$ & & & & & \\
\hline \multicolumn{9}{|l|}{ Body weight } \\
\hline $\mathrm{BMI} \mathrm{kg} \cdot \mathrm{m}^{-2}$ & $27.1 \pm 3.73$ & $26.8 \pm 3.64$ & $27.7 \pm 3.92$ & 0.001 & $27.38 \pm 4.5$ & $27.52 \pm 4.58$ & $27.22 \pm 4.59$ & 0.5 \\
\hline Overweight & $1332(49.6)$ & 957 (48.9) & 375 (51.7) & & 159 (39.8) & $91(38.6)$ & $68(41.5)$ & 0.8 \\
\hline Obese & $533(19.9)$ & 363 (18.5) & $170(23.4)$ & & $110(27.5)$ & $66(28.0)$ & $44(26.8)$ & \\
\hline Systolic blood pressure & $126.9 \pm 16.1$ & $126.3 \pm 15.9$ & $128.6 \pm 16.7$ & 0.001 & & & & \\
\hline AHI & $20.02 \pm 12.7$ & $19.9 \pm 12.4$ & $20.4 \pm 13.6$ & 0.3 & $28.23 \pm 18.7$ & $27.978 .71 \pm 18.8$ & $28.5 \pm 18.8$ & 0.7 \\
\hline \multicolumn{9}{|l|}{ Lifestyle } \\
\hline Current smokers & $53(2.0)$ & $32(1.6)$ & $21(3.0)$ & 0.015 & $5(1.2)$ & $3(1.2)$ & $2(1.3)$ & 1.0 \\
\hline Ex-smokers & 1566 (58.3) & $1124(57.4)$ & $442(60.9)$ & & & & & \\
\hline Current consumers of alcohol & 1750 (65.5) & 1319 (67.7) & 431 (59.7) & $<0.001$ & $147(36.5)$ & 90 (37.2) & $57(34.5)$ & 0.6 \\
\hline \multicolumn{9}{|l|}{ Medical history } \\
\hline Diabetes & 353 (13.2) & $237(12.1)$ & $116(16.0)$ & 0.008 & $51(12.5)$ & $28(11.6)$ & 23 (13.9) & 0.5 \\
\hline $\mathrm{CAD} / \mathrm{MI}$ & $455(17.0)$ & $329(16.8)$ & $126(17.4)$ & 0.7 & $51(12.7)$ & $24(10.0)$ & $27(16.4)$ & 0.06 \\
\hline $\mathrm{CHF}$ & $140(5.2)$ & $93(4.7)$ & $47(6.5)$ & 0.07 & $32(7.9)$ & $18(7.5)$ & $14(8.5)$ & 0.7 \\
\hline Hypertension & 1322 (49.3) & 949 (48.4) & $373(51.4)$ & 0.17 & 239 (58.9) & $148(61.4)$ & $91(55.2)$ & 0.2 \\
\hline Stroke & 100 (3.7) & 68 (3.5) & $32(4.4)$ & 0.25 & 55 (13.5) & 26 (10.8) & $29(17.6)$ & 0.056 \\
\hline Asthma & $211(7.9)$ & $141(7.2)$ & $70(9.6)$ & 0.04 & $49(12.1)$ & 29 (12.0) & $20(12.1)$ & 1.0 \\
\hline COPD & $137(5.1)$ & $83(4.2)$ & $54(7.4)$ & 0.001 & & & & \\
\hline
\end{tabular}

Data are presented as mean \pm SD or $n(\%)$, unless otherwise stated. MrOS: Osteoporotic Fractures in Men Study; SOF: Study of Osteoporotic Fractures; fR: respiratory frequency; PSG: polysomnography; BMI: body mass index; AHI: apnoea-hypopnoea index; CAD: coronary artery disease; MI: myocardial infarction; CHF: congestive heart failure; COPD: chronic obstructive pulmonary disease. 
significantly between dichotomised groups in any of the comparisons. Correlations were assessed using Spearman's rank coefficient.

\section{Results}

\section{Participant characteristics}

Flowcharts of participants included in the study are shown in figure 1. Characteristics of the MrOS Sleep and SOF cohorts are summarised in table 1.

Participants in the MrOS cohort were predominantly white, aged $76 \pm 5$ years and their mean \pm SD body mass index (BMI) was $27.1 \pm 3.7 \mathrm{~kg} \cdot \mathrm{m}^{-2}$ at the baseline visit; $50 \%$ of men were overweight and $20 \%$ were obese. $66 \%$ of men were consuming alcohol; $2 \%$ were current smokers. Nearly $50 \%$ of men reported a history of hypertension; $13.2 \%$ had diabetes; $17.0 \%$ had a history of CAD/myocardial infarction and $5.2 \%$ had heart failure. Histories of asthma and COPD were reported by $7.9 \%$ and $5.1 \%$ of participants, respectively. The incidence of stroke (3.7\%) was low. The average AHI obtained from overnight PSG was $20.0 \pm 12.7$ events $\cdot h^{-1}$.

The SOF cohort comprised only white women, was older at baseline ( $81 \pm 3$ years), but the BMI was comparable $\left(27.4 \pm 4.5 \mathrm{~kg} \cdot \mathrm{m}^{-2}\right) .40 \%$ women were overweight and $28 \%$ were obese. Similar to the male

\section{a)}

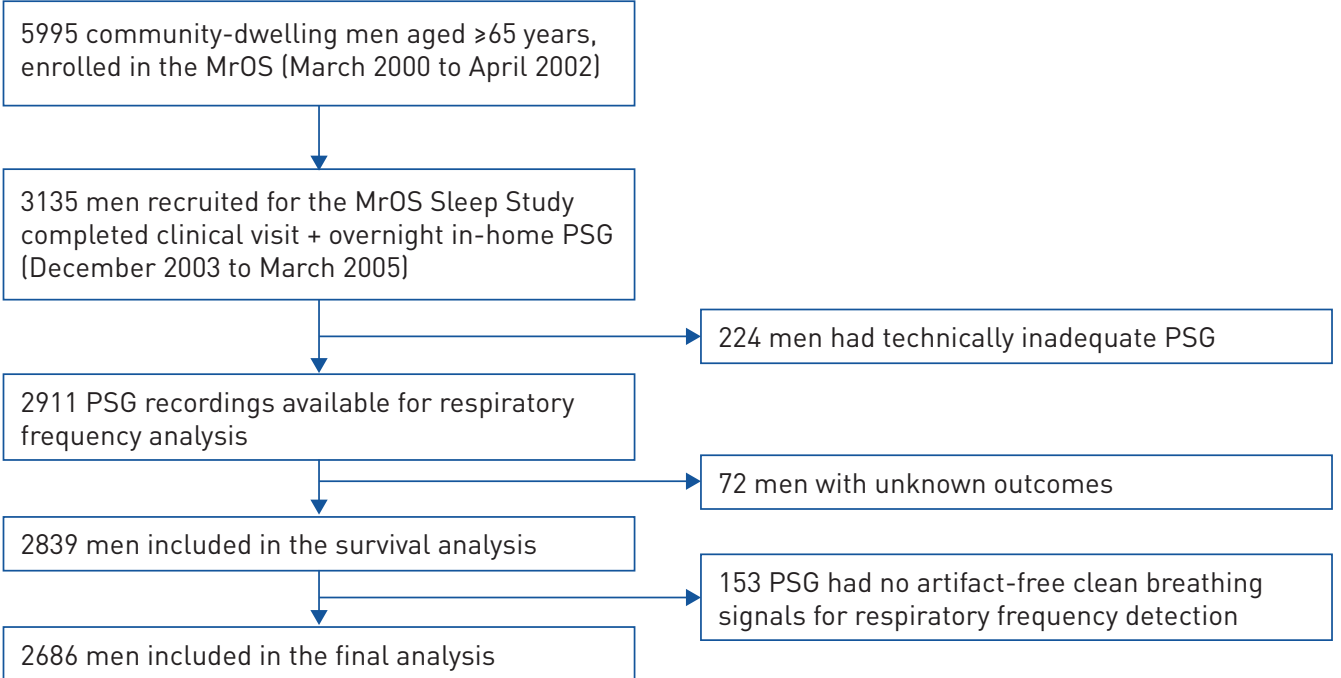

b)

9704 community-dwelling women aged $\geqslant 65$ years enrolled in the SOF (September 1986 to October 1988)

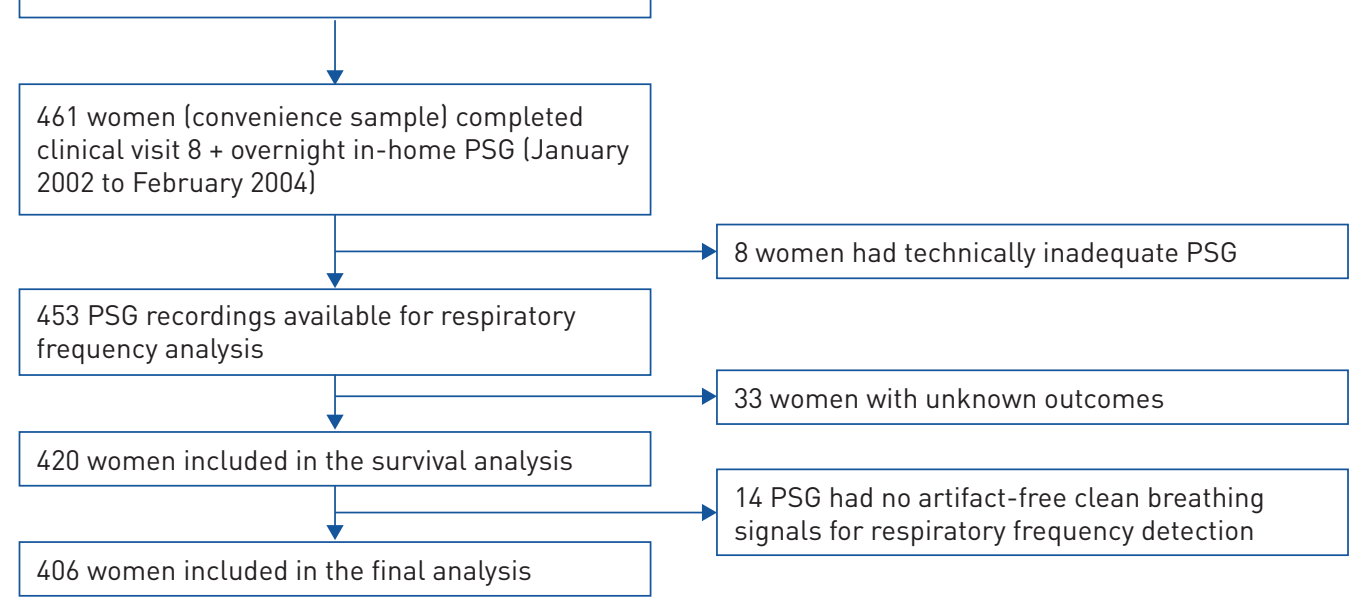

FIGURE 1 Flow charts of participants included in the analysis of respiratory rate for the a) Osteoporotic Fractures in Men Study (MrOS) and b) Study of Osteoporotic Fractures (SOF). PSG: polysomnography. 
cohort, the percentage of current smokers was low (1.2\%), but the proportion of women who consume alcohol was notably lower (37\%). Frequencies of diabetes (13\%), CAD/myocardial infarction (13\%), heart failure (7.9\%) and asthma (12\%) were largely comparable, while the incidence of hypertension (59\%) and stroke $(14 \%)$ was $10 \%$ higher than in the male cohort. History of COPD was not reported in SOF.

Mean nocturnal $f R$ in the MrOS and SOF cohorts were $14.8 \pm 1.8$ breaths $\cdot \mathrm{min}^{-1}$ and $15.5 \pm 1.7$ breaths $\cdot \mathrm{min}^{-1}$, respectively.

\section{Cardiovascular and all-cause mortality}

In the MrOS cohort, outcome data on mortality were available for 2686 participants. During the follow-up period of $8.9 \pm 2.6$ years on average, $166(6.1 \%)$ men died from cardiovascular disease. Among those were 94 confirmed coronary heart disease deaths, 31 cerebrovascular deaths, 21 heart failure deaths, four peripheral vascular disease deaths and four arrhythmic deaths. The follow-up for all-cause mortality continued beyond the detailed tracking of cardiovascular events. During a period of $13.7 \pm 3.7$ years on average, 1312 (51.2\%) out of 2560 men died from any cause.

In the SOF cohort, outcome data on mortality were available for 406 participants. During the $6.4 \pm 1.6$ years of follow-up, $46(11.2 \%)$ women died from cardiovascular disease as defined by the ICD-9 code. Of those, 17 deaths were attributed to ischaemic heart disease, 18 to sudden death post-coronary heart disease, 15 to stroke and 29 to atherosclerosis. 106 (26.1\%) women died from any cause.

\section{Univariate survival analysis}

The Kaplan-Meier curves of nocturnal $f_{R}$ quartiles of both cohorts (figure 2) illustrate significantly elevated cardiovascular mortality in participants whose $f_{R}$ was in the highest quartile (MrOS 16.118.6 breaths $\cdot \mathrm{min}^{-1}$; SOF $16.8-18.6$ breaths $\cdot \mathrm{min}^{-1}$ ) versus those in the lower three quartiles, in whom mortality was largely comparable (10-year mortality in $\operatorname{MrOS} 8.2 \%$ versus $5.3 \%, \mathrm{p}=0.023$; 8-year mortality in SOF: $9.1 \%$ versus $17.6 \%, \mathrm{p}=0.031$ ).

Considering the increased cardiovascular mortality of participants in the highest $f \mathrm{R}$ quartile, we dichotomised both cohorts into subgroups of participants with $f \mathrm{R}<16$ versus $\geqslant 16$ breaths. $\min ^{-1}$ (table 1 ) and explored the association between $f \mathrm{R}$ and participant characteristics in the MrOS and SOF cohorts. MrOS participants with high $f R$ were marginally, yet significantly, older, while the age difference between $f_{\mathrm{R}}$-dichotomised subgroups was not significant in the SOF cohort. Likewise, men with high $f_{\mathrm{R}}$ had marginally but significantly higher BMI, whereas no significant BMI difference between $f$ R subgroups was seen between women. MrOS participants with high $f_{\mathrm{R}}$ were more likely to smoke and less likely to consume alcohol, but this effect was not observed in women. Systolic blood pressure, diabetes, asthma and COPD (not reported in SOF) were positively associated with $f \mathrm{R}$ in men, while hypertension was weakly negatively associated with $f_{R}$ in women. There was no significant association with the prevalence of respiratory events counted by $\mathrm{AHI}$ or cardiac disease and high $f \mathrm{R}$ in either men or women.

Kaplan-Meier curves of all-cause mortality show a significantly higher mortality for men as well as women with $\geqslant 16$ breaths $\cdot \min ^{-1}$ (supplementary material).

\section{Cox-proportional hazard analysis}

Unadjusted Cox-proportional hazard analysis shows significant associations with nocturnal $f \mathrm{R}$ (as a continuous variable) and cardiovascular mortality (table 2). Nocturnal $f R \geqslant 16$ breaths $\cdot$ min $^{-1}$ represented a hazard ratio (HR) of $1.91(\mathrm{p}<0.001)$ in the MrOS cohort and an HR of $2.64(\mathrm{p}=0.002)$ in the SOF cohort.

After adjusting the Cox regression model of nocturnal $f \mathrm{R}$ in MrOS for age, BMI category, smoking, alcohol consumption, diabetes, asthma, systolic blood pressure, AHI and COPD, $f \mathrm{R} \geqslant 16$ breaths.min ${ }^{-1}$ remained a significant predictor of cardiovascular mortality $(\mathrm{HR}=1.57, \mathrm{p}=0.005)$. In the SOF cohort, nocturnal $f_{\mathrm{R}}$ remained a significant predictor of cardiovascular mortality after adjusting for hypertension, AHI and stroke (continuous $f \mathrm{R} H \mathrm{HR}=1.34, \mathrm{p}=0.005$; dichotomised $f \mathrm{R} H \mathrm{H}=2.58, \mathrm{p}=0.002$ ).

When considering all-cause mortality as outcome, univariate as well as adjusted Cox regression models show significant predictive value of $f \mathrm{R}$ when included in the model as a continuous or dichotomised variable (table 2).

\section{Discussion}

This study is the first to demonstrate the prognostic value of nocturnal $f \mathrm{R}$ for long-term cardiovascular and all-cause mortality in older community-dwelling individuals. In two large, independent cohorts of older men and women, nocturnal $f R \geqslant 16$ breaths $\cdot \min ^{-1}$ was significantly and independently associated with 9-year (MrOS) and 6-year (SOF) cardiovascular mortality as well as 14-year (MrOS) and 6-year (SOF) all-cause mortality. 


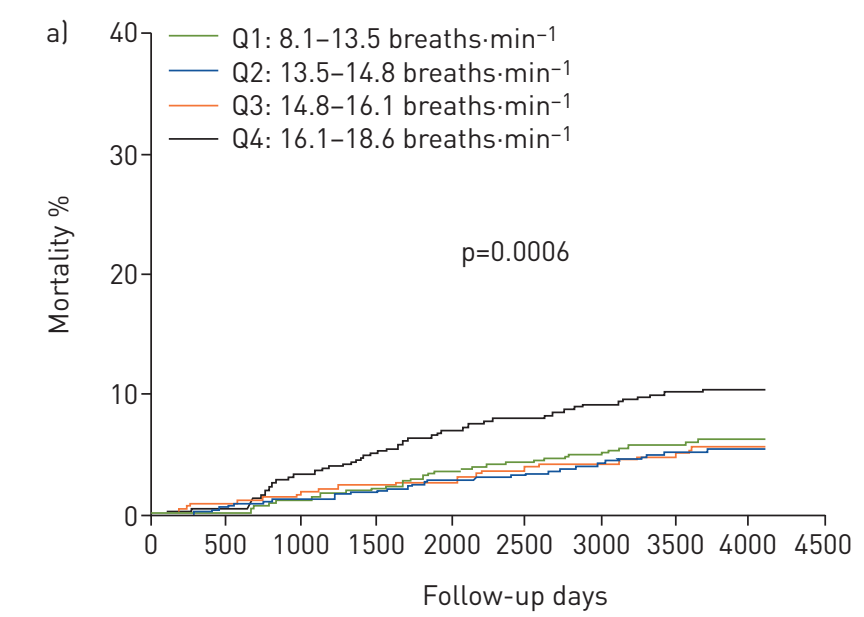

$\begin{array}{llllllllll}\text { At risk n } & 2685 & 2647 & 2551 & 2431 & 2293 & 2149 & 1989 & 1810 & 230\end{array}$ $\begin{array}{llllllllll}\text { Deaths n } & 0 & 16 & 51 & 77 & 104 & 122 & 140 & 159 & 166\end{array}$

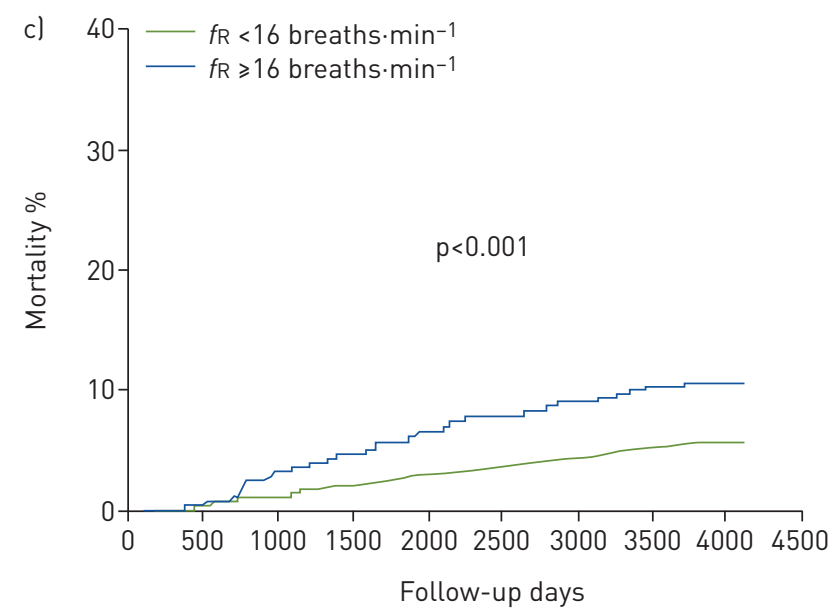

$\begin{array}{llllllllll}\text { At risk n } & 2685 & 2647 & 2551 & 2431 & 2293 & 2149 & 1989 & 1810 & 230\end{array}$ $\begin{array}{llllllllll}\text { Deaths n } & 0 & 16 & 51 & 77 & 104 & 122 & 140 & 159 & 166\end{array}$

FIGURE 2 Cardiovascular mortality analysis of nocturnal respiratory frequency ( $f R$ ). Kaplan-Meier curves for quartiles of $f R$ in al the Osteoporotic Fractures in Men Study (MrOS) Sleep cohort and b) the Study of Osteoporotic Fractures (SOF) cohort and log-rank test results. Cardiovascular mortality for $\mathrm{fR}$ dichotomised at 16 breaths $\mathrm{min}^{-1}$ in $\mathrm{cl}$ the MrOS cohort and d) the SOF cohort. Q1-Q4: quartiles 1-4.

TABLE 2 Association of respiratory frequency $(f R)$ with cardiovascular and all-cause mortality by Cox regression

\begin{tabular}{|c|c|c|c|c|c|c|c|c|}
\hline & \multicolumn{4}{|c|}{ Cardiovascular mortality } & \multicolumn{4}{|c|}{ All-cause mortality } \\
\hline & \multicolumn{2}{|c|}{ Univariate analysis } & \multicolumn{2}{|c|}{ Multivariable analysis" } & \multicolumn{2}{|c|}{ Univariate analysis } & \multicolumn{2}{|c|}{ Multivariable analysis" } \\
\hline & HR $(95 \% \mathrm{CI})$ & p-value & HR $(95 \% \mathrm{CI})$ & p-value & HR $(95 \% \mathrm{CI})$ & p-value & HR $(95 \% \mathrm{CI})$ & p-value \\
\hline \multicolumn{9}{|l|}{ MrOS Sleep } \\
\hline$f_{\mathrm{R}}$ breaths $\cdot \mathrm{min}^{-1}$ & $1.13(1.03-1.23)$ & 0.007 & $1.07(0.98-1.17)$ & 0.14 & $1.10(1.06-1.14)$ & $<0.001$ & $1.04(1.01-1.08)$ & 0.005 \\
\hline$f \mathrm{R} S \mathrm{D}$ & $1.24(1.06-1.45)$ & 0.007 & $1.13(0.96-1.32)$ & 0.14 & $1.19(1.13-1.26)$ & $<0.001$ & $1.08(1.02-1.14)$ & 0.005 \\
\hline$f_{\mathrm{R}}$ breaths $\cdot \mathrm{min}^{-1}$ & $1.36(1.11-1.66)$ & 0.002 & $1.34(1.10-1.65)$ & 0.005 & $1.20(1.06-1.37)$ & 0.004 & $1.20(1.06-1.37)$ & 0.005 \\
\hline$f_{\mathrm{R}} \mathrm{SD}$ & $1.66(1.19-2.31)$ & 0.002 & $1.62(1.16-2.27)$ & 0.005 & $1.35(1.10-1.66)$ & 0.004 & $1.35(1.10-1.67)$ & 0.005 \\
\hline$f_{R} \geqslant 16$ breaths $\cdot \min ^{-1}$ & $2.64(1.44-4.83)$ & 0.002 & $2.58(1.41-4.76)$ & 0.002 & $1.50(1.02-2.19)$ & 0.03 & $1.50(1.02-2.20)$ & 0.04 \\
\hline $\begin{array}{l}\text { MrOS Sleep: Osteopor } \\
\text { category, smoking, al }\end{array}$ & Erostures in & $C$ & tudy; SOF: & 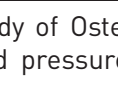 & 5 & $\begin{array}{l}\text { adj } \\
\text { inde }\end{array}$ & $\begin{array}{l}\text { for age, body } \\
\text { I) and chroni }\end{array}$ & $\begin{array}{l}\text { s index } \\
\text { tructive }\end{array}$ \\
\hline
\end{tabular}


The prognostic value of nocturnal mean $f \mathrm{R}$ for long-term mortality may derive from its ability to capture a composite of different factors, including dysfunctional central respiratory control associated with neural disease, and/or compensatory mechanisms for respiratory or metabolic impairments due to diabetes or chronic renal, pulmonary or cardiac failure. In our data, nocturnal mean $f \mathrm{R} \geqslant 16$ breaths.min ${ }^{-1}$ was associated with diabetes, asthma and COPD, but, importantly, demonstrated predictive value beyond these factors. Correlation analysis of nocturnal mean $f \mathrm{R}$ and spirometry, both of which were conducted as part of a MrOS follow-up sleep visit, showed a weak inverse relationship between nocturnal mean $f \mathrm{R}$ and forced expiratory volume in $1 \mathrm{~s}$ and forced vital capacity, but not with the ratio of the latter (data not shown). This suggests that nocturnal mean $f \mathrm{R}$ is in part reflective of daytime respiratory function.

A history of cardiac disease and the severity of concomitant SDB determined by AHI were not significantly associated with $f_{\mathrm{R}} \geqslant 16$ breaths $\mathrm{min}^{-1}$, suggesting that our findings are not solely explained by pre-existing heart failure or SDB as defined by the AHI. While pathophysiological insights cannot be gained and causal relationships cannot be established from cohort studies, elevated $f \mathrm{R}$ is likely to reflect an attempt to restore homeostasis/eucapnia in the presence of impaired lung or airway mechanics [20]. In extreme cases, elevated $f \mathrm{R}$ may cause hypocapnia that can lead to electrolyte abnormalities such as hypokalaemia, cardiac arrhythmias and conduction abnormalities [21], thereby providing a mechanism by which $f \mathrm{R}$ captures cardiovascular mortality risk. Various $f \mathrm{R}$-related and -unrelated organ-specific or more systemic compensatory mechanisms may contribute to increased all-cause mortality in patients with nocturnal $f R \geqslant 16$ breaths $\min ^{-1}$. Additionally, $f R$ during the night may represent a risk marker in a patient group with increased all-cause mortality.

Comparing the MrOS and SOF cohorts, the associations between participant characteristics and nocturnal mean $f \mathrm{R}$ were stronger in the MrOS cohort than in the SOF cohort, but the effect size was generally small. Sex-specific differences in ventilatory control may account for the more pronounced findings in men versus women. For example, hypoxic ventilatory responses are more robust in men than in women [22], which may therefore make them more prone to an elevated $f \mathrm{R}$. The discrepancy in associations between men and women might also be partly attributed to the higher statistical power of the MrOS cohort, where the sample size was more than six times bigger. In addition, the higher age in SOF may have had an impact and possibly contributed to the higher HR observed in women compared to men. Considering the predictive value of $f R$ for cardiovascular versus all-cause mortality in both cohorts, HR were consistently numerically higher for the former.

In the clinical setting, assessment of cardiorespiratory vital parameters, including $f \mathrm{R}$, forms an important part of a standard physiological examination. Although often omitted from anamnesis, our findings make a case for reconsideration of $f R$. Simple, visual inspection of chest movements yields reliable $f R$ measurement with the high inter-observer agreement [23]; alternatively, breathing-related chest movements can be readily automatically obtained from impedance changes derived from ECG Holter recordings [24] or respiratory inductance plethysmography during overnight sleep studies; furthermore, contact-free motion measurement is available via radiofrequency electronics $[25,26]$. Wearable health gadgets and smart watches [27] also allow for simple automated measurement of $f R$ in large parts of the population.

From a measurement point of view, assessing $f \mathrm{R}$ during sleep, as performed here, appears advantageous, because the body is in the supine position at rest in a state of equilibrium, which represents a standardised condition, reducing disturbing factors and noise levels. Future studies need to establish the relationship between daytime $f R$ and nocturnal $f R$. During sleep, tidal volume is reduced, the breathing pattern is shallower, but $f_{\mathrm{R}}$ is slightly increased [28]. Although nocturnal $f_{\mathrm{R}}$ varies between rapid-eye-movement (REM) and non-REM sleep, our analysis yielded similar results (data not shown).

\section{Limitations}

Our observations are based on predominantly white older men and women and cannot be extrapolated to other populations. The age at baseline for men was quite high ( $76 \pm 6$ years) and even more so for women ( $81 \pm 3$ years), which may have introduced a "survivor" bias. Baseline exposure to various conditions was self-reported rather than systematically ascertained through medical records or direct measurement. We did not take into consideration possible confounding effects of medications.

Since nocturnal $f \mathrm{R}$ may be affected by SDB, we excluded episodes of apnoea and hypopnoea from our initial $f R$ measurement, but the main findings remained unaffected when the entire dataset was reanalysed without keeping out various scored (respiratory) events. Our findings are based on single-night overnight PSG; further studies must address intra-individual variability. Additionally, the most effective recording technique (e.g. full PSG or simple ECG) needs to be determined. Prospective studies are warranted to confirm the prognostic value of nocturnal mean $f \mathrm{R}$ as a risk marker in the general population; mechanistic 
and outcome studies should test whether interventions lowering $f \mathrm{R}$ during the night could reduce cardiovascular mortality and whether changes in $f \mathrm{R}$ can help to guide treatment of the underlying disease.

\section{Conclusions}

In community-dwelling older men and women, nocturnal $f \mathrm{R}$ is an independent predictor of long-term cardiovascular and all-cause mortality. Whether $f \mathrm{R}$ could be used as a risk marker for mortality in the clinical setting warrants further prospective studies.

Conflict of interest: M. Baumert has nothing to disclose. D. Linz reports having served on the advisory board of LivaNova, and has received lecture and/or consulting fees from LivaNova and ResMed. K. Stone has nothing to disclose. R.D. McEvoy has nothing to disclose. S. Cummings has nothing to disclose. S. Redline has nothing to disclose. R. Mehra has nothing to disclose. S. Immanuel has nothing to disclose.

Support statement: This study was supported through a grant from the Australian Research Council (DP0663345). The MrOS Study and the SOF Study are supported by National Institutes of Health funding. The following institutes provided support: The National Institute on Aging (NIA), the National Institute of Arthritis and Musculoskeletal and Skin Diseases (NIAMS), the National Center for Advancing Translational Sciences (NCATS), and NIH Roadmap for Medical Research (U01 AG027810, U01 AG042124, U01 AG042139, U01 AG042140, U01 AG042143, U01 AG042145, U01 AG042168, U01 AR066160, and UL1 TR000128). The National Heart, Lung, and Blood Institute (NHLBI) provided funding for the MrOS Sleep ancillary study (R01 HL071194, R01 HL070848, R01 HL070847, R01 HL070842, R01 HL070841, R01 HL070837, R01 HL070838, R01 HL070839) and the National Sleep Research Resource (R24-HL-114473). The SOF sleep study was supported by grant AG021918, AG026720, AG05394, AG05407, AG08415, AR35582, AR35583, AR35584, R01 AG005407, R01 AG027576-22, 2 R01 AG005394-22A1, 2 R01 AG027574-22A1, HL40489 and T32 AG000212-14. S. Redline was supported in part by NIH R35HL135818. The study sponsors had no role in study design; in the collection, analysis, and interpretation of data; in the writing of the report; and in the decision to submit the paper for publication. Funding information for this article has been deposited with the Crossref Funder Registry.

\section{References}

1 Wilson AH, Kidd AC, Skinner J, et al. A simple 5-point scoring system, NaURSE $\left(\mathrm{Na}^{+}\right.$, urea, respiratory rate and shock index in the elderly), predicts in-hospital mortality in oldest old. Age Ageing 2014; 43: 352-357.

2 Shappell C, Snyder A, Edelson DP, et al. Predictors of in-hospital mortality after rapid response team calls in a 274 hospital nationwide sample. Crit Care Med. 2018; 46: 1041-1048.

3 Parkes R. Rate of respiration: the forgotten vital sign. Emerg Nurse 2011; 19: 12-17.

4 Lim WS, Lewis S, Macfarlane JT. Severity prediction rules in community acquired pneumonia: a validation study. Thorax 2000; 55: 219-223.

5 Nagata I, Abe T, Uchida M, et al. Ten-year inhospital mortality trends for patients with trauma in Japan: a multicentre observational study. BMJ Open 2018; 8: e018635.

6 Shah B, Bartaula B, Adhikari J, et al. Predictors of in-hospital mortality of acute ischemic stroke in adult population. J Neurosci Rural Pract 2017; 8: 591-594.

7 Andersen LW, Kim WY, Chase M, et al. The prevalence and significance of abnormal vital signs prior to in-hospital cardiac arrest. Resuscitation 2016; 98: 112-117.

8 Metra M, Cotter G, El-Khorazaty J, et al. Acute heart failure in the elderly: differences in clinical characteristics, outcomes, and prognostic factors in the VERITAS Study. J Card Fail 2015; 21: 179-188.

9 Rodríguez-Molinero A, Narvaiza L, Ruiz J, et al. Normal respiratory rate and peripheral blood oxygen saturation in the elderly population. J Am Geriatr Soc 2013; 61: 2238-2240.

10 Fagard RH, Celis H, Thijs L, et al. Daytime and nighttime blood pressure as predictors of death and cause-specific cardiovascular events in hypertension. Hypertension 2008; 51: 55-61.

11 Kannel WB, Kannel C, Paffenbarger RS, et al. Heart rate and cardiovascular mortality: the Framingham Study. Am Heart J 1987; 113: 1489-1494.

12 Dommasch M, Sinnecker D, Barthel P, et al. Nocturnal respiratory rate predicts non-sudden cardiac death in survivors of acute myocardial infarction. J Am Coll Cardiol 2014; 63: 2432-2433.

13 Barthel P, Wensel R, Bauer A, et al. Respiratory rate predicts outcome after acute myocardial infarction: a prospective cohort study. Eur Heart J 2013; 34: 1644-1650.

14 Heckmann $\mathrm{C}$, van Leeuwen $\mathrm{P}$, Engelke $\mathrm{P}$, et al. Circadian variations of heart rate, respiratory rate and pulse respiration ratio in routinely examined hospital patients. J Interdiscipl Cycle 1990; 21: 198-199.

15 Orwoll E, Blank JB, Barrett-Connor E, et al. Design and baseline characteristics of the osteoporotic fractures in men (MrOS) study- a large observational study of the determinants of fracture in older men. Contemp Clin Trials 2005; 26: 569-585.

16 Blank JB, Cawthon PM, Carrion-Petersen ML, et al. Overview of recruitment for the osteoporotic fractures in men study (MrOS). Contemp Clin Trials 2005; 26: 557-568.

17 Cummings SR, Black DM, Nevitt MC, et al. Appendicular bone density and age predict hip fracture in women. The Study of Osteoporotic Fractures Research Group. JAMA 1990; 263: 665-668.

18 Spira AP, Blackwell T, Stone KL, et al. Sleep-disordered breathing and cognition in older women. J Am Geriatr Soc 2008; 56: 45-50.

19 Koo BB, Blackwell T, Ancoli-Israel S, et al. Association of incident cardiovascular disease with periodic limb movements during sleep in older men: outcomes of sleep disorders in older men (MrOS) study. Circulation 2011; 124: $1223-1231$.

20 Henke KG, Dempsey JA, Kowitz JM, et al. Effects of sleep-induced increases in upper airway resistance on ventilation. J Appl Physiol 1990; 69: 617-624. 
21 Javaheri S, Corbett W. Association of low $\mathrm{PaCO}_{2}$ with central sleep apnea and ventricular arrhythmias in ambulatory patients with stable heart failure. Ann Intern Med 1998; 128: 204-207.

22 Douglas NJ. Control of ventilation during sleep. Clin Chest Med 1985; 6: 563-575.

23 Nielsen LG, Folkestad L, Brodersen JB, et al. Inter-observer agreement in measuring respiratory rate. PLoS One 2015; 10: e0129493.

24 Sinnecker D, Dommasch M, Barthel P, et al. Assessment of mean respiratory rate from ECG recordings for risk stratification after myocardial infarction. J Electrocardiol 2014; 47: 700-704.

25 Droitcour A, Lubecke V, Lin J, et al. A microwave radio for Doppler radar sensing of vital signs. IEEE MTTS Int Microwave Symp Dig 2001; 1: 175-178.

26 Ballal T, Heneghan C, Zaffaroni A, et al. A pilot study of the nocturnal respiration rates in COPD patients in the home environment using a non-contact biomotion sensor. Physiol Meas 2014; 35: 2513-2527.

27 Appelboom G, Camacho E, Abraham ME, et al. Smart wearable body sensors for patient self-assessment and monitoring. Arch Public Health 2014; 72: 28.

28 Douglas NJ, White DP, Pickett CK, et al. Respiration during sleep in normal man. Thorax 1982; 37: 840-844. 